\title{
9 PREVALÊNCIA DE TRANSTORNOS MENTAIS COMUNS EM ADULTOS NO CONTEXTO DA ATENÇÃO PRIMÁRIA À SAÚDE
}

\author{
| Luís Souza e Souza ${ }^{1}$; Bruna Barbosa ${ }^{2}$; Carla de Oliveira e Silva ${ }^{3}$; Antônia de Souza ${ }^{4}$ Tadeu Ferreira ${ }^{5}$, Leila Siqueira ${ }^{6} \mid$
}

\section{RESUMO}

CONTEXTO: Os transtornos mentais comuns (TMC) abrangem sintomas de fadiga, esquecimento, irritabilidade, dificuldades de concentração, queixas somáticas e sentimento de inutilidade. Altas prevalências destes agravos têm sido observadas em adultos no contexto da Atenção Primária, sendo consideradas uma das maiores problemáticas de saúde no mundo.

OBJETIVO: Estimar a prevalência e fatores associados aos TMC em adultos assistidos por uma equipe da Estratégia Saúde da Família no Brasil.

MÉTODOS: Estudo transversal e analítico, realizado no ano 2016, com uma amostra aleatória de 297 adultos. Para coleta dos dados, utilizou-se o questionário Self-Reporting Questionnaire (SRQ-20), além de informações sociodemográficas, sobre condições de vida e saúde. Para presença de TMC, considerou-se ponto de corte o escore igual ou maior que 7 para homens e mulheres. Testaram-se as associações dos TMC com as variáveis de interesse, por meio de análise univariada (qui-quadrado), com nível de significância de 5\%.

RESULTADOS: A prevalência dos TMC foi de 23,2\%. A prevalência do desfecho foi significativamente maior nos adultos do sexo feminino, em união estável, com 0 a 4 anos de estudos, que faziam uso de medicamento controlado, e que informaram diagnóstico prévio/confirmado de TMC pessoal ou na família.

CONCLUSÕES: Os resultados apresentados evidenciam que as características associadas são passíveis de serem investigadas e acompanhadas na prática clínica. Destaca-se a necessidade de qualificação dos profissionais da Atenção Primária à Saúde, visando à detecção e acolhimento das pessoas com TMC, com enfoque em uma assistência integral e que envolva a família.

\section{PALAVRAS-CHAVE: Transtornos mentais; Atenção primária à saúde; Assistência à saúde mental}

\section{RESUMEN}

\section{"Prevalencia de trastornos mentales comunes en adultos en el contexto de la Atención Primaria de Salud"}

CONTEXTO: Los trastornos mentales comunes (TMC) cubren los síntomas de fatiga, falta de memoria, irritabilidad, dificultades de concentración, quejas somáticas y sentimientos de inutilidad. Se han observado en adultos en contexto de atención primaria, siendo considerado un daos de problema de salud más importantes del mundo.

OBJETIVO: Estimar la prevalencia y factores asociados a TMC en adultos asistidos por un equipo de la Estrategia de Salud de la Familia en Brasil. METODOLOGÍA: Estudio transversal y analítico, conducido en el año 2016, con una muestra aleatoria de 297 adultos. Para la recolección de datos, se utilizó el cuestionario Self-Reporting Questionnaire (SRQ-20), así como información sociodemográfica sobre las condiciones de vida y la salud. Para establecer la TMC, se consideró la puntuación igual o superior a 7 para hombres y mujeres. Las asociaciones fueron probados TMC con las variables de interés, a través de análisis univariado (Chi-cuadrado), con un nivel de significancia del 5\%.

RESULTADOS: La prevalencia de TMC fue 23,2\%. La prevalencia del evento fue significativamente mayor en mujeres adultas, en establo, con 0 a 4 años de estudio, que hacen uso de drogas controladas, y con diagnóstico previo y confirmado de TMC individualmente o en família.

CONCLUSIONES: Los resultados presentados muestran que las características asociadas suelen ser investigados y seguidos en la práctica clínica. Destaca la necesidad de capacitación de profesionales de Salud Primaria, dirigidas a la detección y recepción de las personas con TMC, centrándose en una asistencia integral y participación de la familia.

\section{DESCRIPTORES: Trastornos mentales; Atención primaria de salud; Atención a la salud mental}

\begin{abstract}
"Prevalence of common mental disorders in adults in the context of Primary Health Care"

BACKGROUND: The common mental disorders (CMD) cover symptoms of fatigue, forgetfulness, irritability, concentration difficulties, somatic complaints and feelings of worthlessness. Has been observed high prevalence of these diseases in adults in adults in the context of Primary Health Care, being considered one of the biggest health problems in the world. AIM: Estimate the prevalence and factors associated with CMD in adults assisted by a team from the Family Health Strategy in Brazil.

METHODS: Cross-sectional study and analytical, performing in the year 2016, with a random sample of 297 adults. For data collection, we used the questionnaire Self-Reporting Questionnaire (SRQ-20), as well as sociodemographic information on living conditions and health. For presence of CMD, cut-off score equal to or greater than 7 for men and women. Associations between the CMD with interest variables were tested, through univariate analysis (Chi-square), with a significance level of $5 \%$.

RESULTS: The prevalence of CMD was $23.2 \%$. The prevalence of the event was significantly higher in female adults, in stable, with 0 to 4 years of study, that made use of controlled drug, and that reported prior diagnosis or confirmed of CMD individual or in the family.

CONCLUSIONS: The results presented show that the associated features are likely to be investigated and followed up in clinical practice. Highlights the need for qualification of Primary Health Care professionals, aimed at the detection and reception of the people with CMD, focusing on a comprehensive assistance and involving the family.
\end{abstract}

\section{KEYWORDS: Mental disorders; Primary health care; Mental health assistance}

Submetido em 22-03-2017

Aceite em 17-11-2017

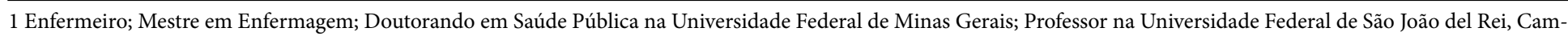
pus Dom Bosco, Departamento de Medicina, Praça Dom Helvécio, 74, 36301-160 São João del Rei, Minas Gerais, Brasil, luis.pauloss@hotmail.com

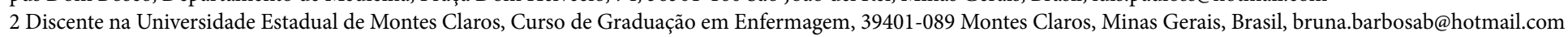

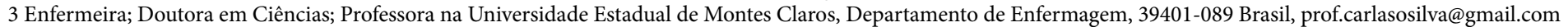
4 Discente nas Faculdades Integradas Pitágoras de Montes Claros, Curso de Graduação em Psicologia, 39408-007 Minas Gerais, Brasil, antoniagoncalves8@gmail.com

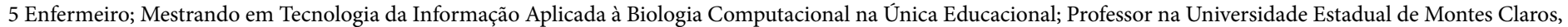
Departamento de Enfermagem, 39401-089 Montes Claros, Minas Gerais, Brasil, tadeu.nunes@hotmail.com

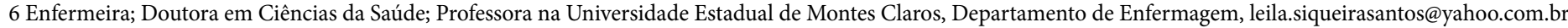

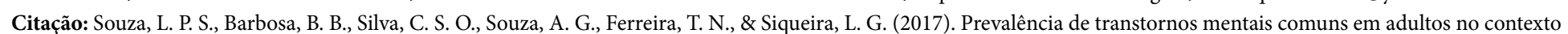
da Atenção Primária de Saúde. Revista Portuguesa de Enfermagem de Saúde Mental (18), 59-66. doi: 10.19131/rpesm.0193 


\section{INTRODUÇÃO}

Os transtornos mentais comuns (TMC) compreendem sintomas como insônia, fadiga, esquecimento, irritabilidade, dificuldades de concentração, queixas somáticas e sentimento de inutilidade (Morais \& Segri, 2011; Portugal, Campos, Gonçalves, Mari \& Fortes, 2016). A denominação de TMC se refere a transtornos mentais menos rigorosos, sendo, às vezes, difusos e inespecíficos em comparação com outros quadros mais definidos pelos sistemas classificatórios e diagnósticos vigentes. São, também, menos distintos e socialmente menos perturbadores e, por isso, seu impacto e prevalência têm recebido pouca atenção por parte das políticas públicas e, consequentemente, do sistema de saúde, mais especificamente na Atenção Primária à Saúde (Morais \& Segri, 2011).

Os TMC possuem significativa prevalência nas sociedades modernas, afetando pessoas de diversas faixas etárias, com destaque para os adultos, pois é nesta fase que, geralmente, iniciam-se as responsabilidades, o que acarreta sofrimento tanto para o indivíduo, quanto para a família e comunidade (Rocha, Almeida, Araújo \& Virtuoso, 2010). Tanto no contexto nacional quanto no internacional, reconhece-se que diversos fatores podem acarretar baixa autoestima, aumentando assim a suscetibilidade de transtornos mentais comuns, tais como: eventos de vida estressantes e desempenho insatisfatório de papéis sociais; problemas interpessoais; internações hospitalares; problemas de saúde; acesso desigual aos cuidados de saúde; estar desempregado ou não ter nenhuma ocupação; condições inadequadas de habitação; abuso do tabaco e do álcool; sedentarismo; ser do sexo feminino; ser imigrante; baixo nível de escolaridade; vítimas de violência (Fonseca, Guimarães \& Vasconcelos, 2008; Gonçalves \& Kapczinski, 2008; Silva et al., 2010; Chandra \& Satyanarayana, 2010; Jenkins, Mbatia, Singleton \& White, 2010; Rocha et al., 2011; Jansen et al., 2011; Coutinho, Rodrigues \& Ramos, 2012; Coutinho et al., 2012; Franken, Coutinho \& Ramos, 2012; Lucchese, Bonfin, Vera \& Santana, 2014).

No Brasil, a prevalência de TMC na população geral é de 29,9\%, chegando a 38\% nos usuários da Atenção Primária à Saúde (APS) (Gonçalves, Tetelbon \& Kapczinski, 2008; Rocha et al., 2010). No contexto mundial, as projeções para 2030 são no sentido de incluírem estas perturbações entres as mais incapacitantes do ser humano (Skapinakis et al., 2013). Estes dados justificam a relevância de medidas de rastreamento dos grupos vulneráveis para o desenvolvimento do TMC na população geral, sobretudo, no âmbito da APS. A investigação de possíveis casos de TMC neste nível de atenção à saúde é um grande problema enfrentado na atualidade, uma vez que os sintomas nem sempre são valorizados. Esta lacuna na identificação por parte dos profissionais da APS compromete a saúde das populações e representa elevado ônus para a saúde pública, tendo em vista que se espera que este nível de atenção rompa com o modelo médico hegemônico, englobe práticas de saúde mental, envolva a família em sua dimensão sociocultural como objeto de atenção, planeje, construa e execute tecnologias para melhoria da qualidade de vida das pessoas nas dimensões bio-psico-socio-espirituais (Rocha et al., 2010; Portugal et al., 2016; Murcho, Pacheco \& Jesus, 2016).

Embora seja assegurado à população o acesso aos cuidados primários e à melhoria de sua qualidade de vida, principalmente quando relacionam as articulações entre Estratégia Saúde da Família (ESF) e atenção à saúde mental, observam-se limitações no rastreamento dos casos de TMC. Assim, identificar a presença destes agravos e dos seus fatores de risco na comunidade torna os profissionais da APS aptos para buscarem meios de atenuar ou eliminar as dificuldades e prestar melhor assistência a esta população (Pie et al., 2013; Lucchese et al., 2014). Torna-se importante que as equipes da ESF estejam atentas aos aspectos da saúde mental da população atendida, para que realizem, efetivamente, a abordagem biopsicossocial, concretizando o papel da APS de reorganização do sistema de atenção à saúde, por meio uma abordagem multidisciplinar e integrada à comunidade que atende, visando ações de educação em saúde, promoção da saúde mental, prevenção de agravos relacionados aos transtornos mentais por meio de intervenção precoce (Gonçalves \& Kapczinski, 2008). Neste sentido, a presente investigação objetivou estimar a prevalência e fatores associados aos TMC em adultos assistidos por uma equipe da Estratégia Saúde da Família de um município da região Sudeste do Brasil.

\section{MÉTODOS}

Estudo transversal e analítico, desenvolvido com adultos cadastrados em uma equipe de Estratégia Saúde da Família no município de Montes Claros, estado de Minas Gerais, região Sudeste do Brasil. A ESF em estudo possuía uma população de 2.527 habitantes em 2014, sendo que destes, 1.492 eram adultos com idade entre 18 a 59 anos. Entre os adultos, 53,5\% eram mulheres e $46,5 \%$ homens. 
A população elegível para este estudo foram os adultos entre 18 e 59 anos de idade que possuíam cadastro na ESF escolhida. Realizou-se o cálculo de amostragem probabilística por meio da equação para estudos de proporção com população finita (Pagano \& Gauvreau, 2011), obtendo amostra estimada de 297 adultos. A amostra foi estratificada por sexo, pelo fato de outros estudos relatarem risco significativamente maior para o TMC no sexo feminino. A amostra foi dividida de forma proporcional entre todas as seis microáreas, sendo, em média, 22 homens e 27 mulheres em cada microárea. Os participantes foram identificados de forma aleatória e convidados a participar da pesquisa, desde que atendessem aos seguintes critérios de inclusão: ser cadastrado na Estratégia Saúde da Família indicada; ter idade entre 18 a 59 anos; não apresentar déficit de comunicação, deficiência mental, transtorno mental severo ou estar sob efeito de álcool e/ou drogas (Carlotto, Amazarray, Chinazzo \& Taborda, 2011; Pie et al., 2013). A coleta de dados foi realizada no domicílio do entrevistado, no período de agosto de 2015 a março de 2016, utilizando o instrumento Self-Reporting Questionnaire (SRQ-20). Este instrumento foi validado no Brasil em 1986, tendo sido readequado como ponto de corte para rastreamento dos TMC na comunidade em 2008. Apresenta uma escala dicotômica ( $\operatorname{sim} /$ não) para cada uma das suas questões, em que cada resposta "sim" equivale a um ponto. O SRQ-20 é considerado como teste de triagem adequado para estudos de populações, sendo muito útil para uma primeira classificação de possíveis casos e não casos (Santos, Araújo, Pinho \& Silva, 2010). Como resultado, observa-se variação entre 0 (nenhuma probabilidade para TMC) a 20 (extrema probabilidade para TMC).

Neste estudo, o ponto de corte utilizado foi o escore de valor igual ou maior que sete (07) para homens e mulheres, conforme recomendado por outros estudos (Gluloum, Bener \& Abou-Salen, 2011; Pie et al., 2013). Além disso, coletaram-se informações sociodemográficas, de condições de vida e de saúde, com instrumento construído pela equipe de pesquisadores, tendo sido embasados por instrumentos de outros estudos que avaliaram TMC em adultos (Rocha et al., 2010; Carlotto et al., 2011; Pie et al., 2013).

A tabulação dos dados foi realizada pelo programa Excel for Windows (2003-2007), após dupla conferência. A análise descritiva dos dados foi obtida por medidas de frequência absoluta e relativa, utilizando o Minitab Statistical Software versão 20.
Para análise de associação univariada, considerou-se como desfecho a pontuação obtida pelo escore maior ou igual a sete (presença de TMC); e como variáveis explicativas, as sociodemográficas, condições de vida e condições de saúde. O teste utilizado foi o Qui-Quadrado de Pearson, com nível de significância de 5\%.

O estudo foi aprovado pelo Comitê de Ética em Pesquisa da Universidade Estadual de Montes Claros Brasil, por meio do parecer consubstanciado número 1.145.395, de 10 de julho de 2015. Todos os participantes assinaram o Termo de Consentimento Livre e Esclarecido (TCLE).

\section{RESULTADOS}

Observou-se que a prevalência de transtorno mental comum na amostra de adultos pesquisada foi de $23,2 \%$. A Tabela 1 mostra os dados sociodemográficos dos 297 adultos, segundo presença ou não de transtorno mental comum.

Tabela 1 - Variáveis Sociodemográficas Segundo Presença ou Não de Transtornos Mentais Comuns

\begin{tabular}{|c|c|c|c|}
\hline Variáveis & $\mathbf{n}(\%)$ & $\begin{array}{c}<7 \text { SRQ-20 } \\
\mathbf{n}(\%)\end{array}$ & $\begin{array}{c}\geq 7 \text { SRQ-20 } \\
\mathbf{n}(\%)\end{array}$ \\
\hline \multicolumn{4}{|l|}{ Sexo } \\
\hline Feminino & $163(54,88)$ & $113(69,33)$ & $50(30,67)$ \\
\hline Masculino & $134(45,12)$ & $115(85,82)$ & $19(14,18)$ \\
\hline \multicolumn{4}{|l|}{ Idade } \\
\hline $18-29$ & $115(38,72)$ & $91(79,13)$ & $24(20,87)$ \\
\hline 50 a 59 & $70(23,57)$ & $51(72,86)$ & $19(27,14)$ \\
\hline 30 a 39 & $64(21,55)$ & $52(81,25)$ & $12(18,75)$ \\
\hline 40 a 49 & $48(16,16)$ & $34(70,83)$ & $14(29,17)$ \\
\hline \multicolumn{4}{|l|}{ Religião } \\
\hline Sim, praticante & $206(69,36)$ & $159(77,18)$ & $47(22,82)$ \\
\hline Sim, não praticante & $47(15,82)$ & $36(76,60)$ & $11(23,40)$ \\
\hline Não & $44(14,82)$ & $33(75,00)$ & $11(25,00)$ \\
\hline \multicolumn{4}{|l|}{ Estado Civil } \\
\hline União Estável & $15(5,05)$ & $7(46,67)$ & $8(53,33)$ \\
\hline Casado(a) & $130(43,77)$ & $107(82,31)$ & $23(17,69)$ \\
\hline Separado(a) & $10(3,37)$ & $5(50,00)$ & $5(50,00)$ \\
\hline Viúvo(a) & $17(5,72)$ & $10(58,82)$ & $7(41,18)$ \\
\hline Solteiro(a) & $125(42,09)$ & $99(79,20)$ & $26(20,80)$ \\
\hline \multicolumn{4}{|l|}{ Cônjuge Falecido } \\
\hline Não & $271(91,25)$ & $210(77,49)$ & $61(22,51)$ \\
\hline $\operatorname{Sim}(<5$ anos $)$ & $17(5,72)$ & $12(70,59)$ & $5(29,41)$ \\
\hline $\operatorname{Sim}(>5$ anos $)$ & $9(3,03)$ & $6(66,67)$ & $3(33,33)$ \\
\hline \multicolumn{4}{|l|}{ Filhos } \\
\hline Sim & $272(91,58)$ & $209(76,84)$ & $63(23,16)$ \\
\hline Não & $25(8,42)$ & $19(76,00)$ & $6(24,00)$ \\
\hline
\end{tabular}




\begin{tabular}{|c|c|c|c|}
\hline \multicolumn{4}{|l|}{ Número de filhos } \\
\hline Nenhum & $110(37,04)$ & $86(78,18)$ & $24(21,82)$ \\
\hline 1 a 3 & $140(47,14)$ & $113(80,71)$ & $27(19,29)$ \\
\hline 4 a 6 & $32(10,77)$ & $19(59,38)$ & $13(40,62)$ \\
\hline 7 ou mais & $15(5,05)$ & $10(66,67)$ & $5(33,33)$ \\
\hline \multicolumn{4}{|l|}{ Anos de estudos } \\
\hline 0 a 4 & $64(21,55)$ & $35(54,69)$ & $29(45,31)$ \\
\hline 5 a 8 & $68(22,90)$ & $58(85,29)$ & $10(14,71)$ \\
\hline 9 ou mais & $165(55,56)$ & $135(81,82)$ & $30(18,18)$ \\
\hline \multicolumn{4}{|c|}{ Atividade Remunerada } \\
\hline Nenhuma & $88(29,63)$ & $64(72,73)$ & $24(27,27)$ \\
\hline $\begin{array}{l}\text { Trabalho com } \\
\text { carteira assinada }\end{array}$ & $77(25,93)$ & $65(84,42)$ & $12(15,58)$ \\
\hline Autônomo(a) & $65(21,89)$ & $52(80,00)$ & $13(20,00)$ \\
\hline $\begin{array}{l}\text { Trabalho sem } \\
\text { carteira assinada }\end{array}$ & $33(11,11)$ & $23(69,70)$ & $10(30,30)$ \\
\hline $\begin{array}{l}\text { Aposentado(a)/ } \\
\text { Afastado(a) }\end{array}$ & $22(7,40)$ & $13(59,09)$ & $9(40,91)$ \\
\hline Vínculo Público & $11(3,70)$ & $10(90,91)$ & $1(9,09)$ \\
\hline Estágio & $1(0,34)$ & $1(100,00)$ & $0(0,00)$ \\
\hline
\end{tabular}

Maior parte da amostra era do sexo feminino (54,88\%); com idade entre 18 e 29 anos (38,72\%); com religião praticante $(69,36 \%)$; casada $(43,77 \%)$; com filhos (91,58\%), sendo que $47,14 \%$ afirmaram ter de 1 a 3 filhos. Além disso, 55,56\% possuíam nível de escolaridade com nove ou mais anos de estudo; 29,63\% afirmaram não exercer nenhuma atividade remunerada.

Já a Tabela 2 mostra os dados relacionados às condições de vida dos participantes, separados entre aqueles que apresentaram transtorno mental comum e entre os que não apresentaram.

Tabela 2 - Variáveis Relacionadas às Condições de Vida Segundo Presença ou Não de Transtornos Mentais Comuns

\begin{tabular}{|c|c|c|c|}
\hline Variáveis & $\mathrm{n}(\%)$ & $\begin{array}{c}<7 \text { SRQ-20 } \\
\mathbf{n}(\%)\end{array}$ & $\begin{array}{c}\geq 7 \text { SRQ-20 } \\
\mathbf{n}(\%)\end{array}$ \\
\hline \multicolumn{4}{|c|}{ Provedor(a) da Família } \\
\hline Não & $122(41,08)$ & $89(72,95)$ & $33(27,05)$ \\
\hline Total & $88(29,63)$ & $71(80,68)$ & $17(19,32)$ \\
\hline Parcial & $87(29,29)$ & $68(78,16)$ & $19(21,84)$ \\
\hline \multicolumn{4}{|c|}{ Renda Mensal Individual } \\
\hline $\begin{array}{l}\text { Até } 1 \text { salário } \\
\text { mínimo }\end{array}$ & $135(45,45)$ & $101(74,81)$ & $34(25,19)$ \\
\hline $\begin{array}{l}2 \text { a } 3 \text { salários míni- } \\
\text { mos }\end{array}$ & $65(21,88)$ & $55(84,62)$ & $10(15,38)$ \\
\hline $\begin{array}{l}4 \text { a } 5 \text { salários míni- } \\
\text { mos }\end{array}$ & $4(1,35)$ & $4(100,00)$ & $0(0,00)$ \\
\hline $\begin{array}{l}6 \text { ou mais salários } \\
\text { mínimos }\end{array}$ & $4(1,35)$ & $3(75,00)$ & $1(25,00)$ \\
\hline Nenhuma & $89(29,97)$ & $65(73,03)$ & $24(26,97)$ \\
\hline
\end{tabular}

\begin{tabular}{|c|c|c|c|}
\hline \multicolumn{4}{|c|}{ Renda Mensal Familiar } \\
\hline $\begin{array}{l}\text { Até } 1 \text { salário } \\
\text { mínimo }\end{array}$ & $125(42,08)$ & $90(72,00)$ & $35(28,00)$ \\
\hline $\begin{array}{l}2 \text { a } 3 \text { salários míni- } \\
\text { mos }\end{array}$ & $140(47,13)$ & $111(79,29)$ & $29(20,71)$ \\
\hline $\begin{array}{l}4 \text { a } 5 \text { salários míni- } \\
\text { mos }\end{array}$ & $18(6,07)$ & $15(83,33)$ & $3(16,67)$ \\
\hline $\begin{array}{l}6 \text { a } 8 \text { salários míni- } \\
\text { mos }\end{array}$ & $12(4,04)$ & $10(83,33)$ & $3(16,67)$ \\
\hline $\begin{array}{l}9 \text { ou mais salários } \\
\text { mínimos }\end{array}$ & $1(0,34)$ & $1(100,00)$ & $0(0,00)$ \\
\hline Nenhuma & $1(0,34)$ & $1(100,00)$ & $0(0,00)$ \\
\hline \multicolumn{4}{|l|}{ Habitação } \\
\hline Própria & $258(86,86)$ & $197(76,36)$ & $61(23,64)$ \\
\hline Alugada & $22(7,40)$ & $19(86,36)$ & $3(13,64)$ \\
\hline Cedida & $16(5,40)$ & $11(68,75)$ & $5(31.25)$ \\
\hline Financiada & $1(0,34)$ & $1(100,00)$ & $0(0,00)$ \\
\hline \multicolumn{4}{|c|}{ Número de pessoas na residência } \\
\hline 1 & $14(4,71)$ & $9(64,29)$ & $5(35,71)$ \\
\hline 2 a 3 & $102(34,34)$ & $80(78,43)$ & $22(21,57)$ \\
\hline 4 a 5 & $122(41,08)$ & $87(71,31)$ & $35(28,69)$ \\
\hline 6 ou mais & $59(19,87)$ & $52(88,14)$ & $7(11,86)$ \\
\hline \multicolumn{4}{|l|}{ Destino dos Dejetos } \\
\hline Esgoto & $296(99,66)$ & $227(76,69)$ & $69(23,31)$ \\
\hline Fossa & $1(0,34)$ & $1(100,00)$ & $0(0,00)$ \\
\hline \multicolumn{4}{|l|}{ Energia Elétrica } \\
\hline Sim & $296(99,66)$ & $227(76,69)$ & $69(23,31)$ \\
\hline Não & $1(0,34)$ & $1(100,00)$ & $0(0,00)$ \\
\hline
\end{tabular}

Foi possível observar que $45,45 \%$ dos participantes informaram renda individual mensal de até um salário mínimo; 47,13\% informaram renda familiar mensal de um a três salários mínimos. Em relação às condições de moradia, maioria $(86,86 \%)$ possuía casa própria, com acesso à energia elétrica $(99,66 \%)$ e rede de esgoto $(99,66 \%)$. As famílias eram compostas, em sua maioria, por quatro a cinco moradores $(41,08 \%)$.

Entre os que apresentaram TMC $(n=69)$, maior parte era do sexo feminino; adulta jovem (18-29 anos de idade); com religião praticante; solteira; não possuíam cônjuge falecido; tinha filhos; tinha 9 ou mais anos de estudo; sem atividade remunerada; com renda individual mensal de até 1 salário mínimo; habitação própria; acesso à rede de esgoto e energia elétrica.

Já a Tabela 3 mostra os dados relativos às condições de saúde dos 297 adultos, segundo presença ou não de transtorno mental comum. 
Tabela 3 - Variáveis Relacionadas às Condições de Saúde Segundo Presença ou Não de Transtornos Mentais Comuns

\begin{tabular}{|c|c|c|c|}
\hline Variáveis & $\mathbf{n}(\%)$ & $\begin{array}{c}<7 \text { SRQ-20 } \\
\text { n(\%) }\end{array}$ & $\begin{array}{l}\geq 7 \text { SRQ-20 } \\
\mathbf{n}(\%)\end{array}$ \\
\hline \multicolumn{4}{|l|}{ Plano de Saúde } \\
\hline Sim & $82(27,61)$ & $65(79,27)$ & $17(20,73)$ \\
\hline Não & $215(72,39)$ & $163(75,81)$ & $52(24,19)$ \\
\hline \multicolumn{4}{|l|}{ Doença Crônica } \\
\hline Não & $240(80,80)$ & $190(79,17)$ & $50(20,83)$ \\
\hline Sim, Hipertensão & $35(11,78)$ & $24(68,57)$ & $11(31,43)$ \\
\hline Sim, Diabetes & $9(3,05)$ & $8(88,89)$ & $1(11,11)$ \\
\hline $\begin{array}{l}\text { Sim, Hipertensão e } \\
\text { Diabetes }\end{array}$ & $10(3,36)$ & $4(40,00)$ & $6(60,00)$ \\
\hline $\begin{array}{l}\text { Sim, Hipotireoid- } \\
\text { ismo }\end{array}$ & $2(0,67)$ & $1(50,00)$ & $1(50,00)$ \\
\hline Sim, Esquizofrenia & $1(0,34)$ & $1(100,00)$ & $0(0,00)$ \\
\hline \multicolumn{4}{|c|}{ Uso de medicamento controlado ${ }^{\star *}$} \\
\hline Sim & $63(21,21)$ & $39(61,90)$ & $24(38,10)$ \\
\hline Não & $234(78,79)$ & $189(80,77)$ & $45(19,23)$ \\
\hline \multicolumn{4}{|c|}{$\begin{array}{l}\text { Diagnóstico prévio/confirmado de TMC no indivíduo ou } \\
\text { alguém na família }\end{array}$} \\
\hline Sim & $59(19,87)$ & $34(57,63)$ & $25(42,37)$ \\
\hline Não & $238(80,13)$ & $194(81,51)$ & $44(18,49)$ \\
\hline \multicolumn{4}{|c|}{$\begin{array}{l}\text { Uso de álcool e outras drogas do indivíduo e/ou alguém na } \\
\text { família }\end{array}$} \\
\hline Sim & $90(30,30)$ & $67(74,44)$ & $23(25,56)$ \\
\hline Não & $207(69,70)$ & $161(77,78)$ & $46(22,22)$ \\
\hline
\end{tabular}

**Observação: Medicamentos controlados são aqueles comercializados apenas mediante receituário médico ou de outro profissional habilitado pela legislação do país. No Brasil, somente poderão prescrever estes medicamentos os profissionais inscritos no Conselho Regional de Medicina ou no Conselho Regional de Medicina Veterinária ou no Conselho Regional de Odontologia. Estes medicamentos são submetidos a um regime especial de controle pelas autoridades e são divididos em entorpecentes, psicotrópicos, retinóides de uso sistêmico e imunossupressores (Ministério da Saúde, 1998).

Como observado, maioria dos adultos não possuía plano de saúde (72,39\%); não fazia uso de medicamentos controlados (78,79\%); não apresentava doenças crônicas $(80,80 \%)$. Além disso, $80,13 \%$ dos pesquisados negaram diagnóstico confirmado de TMC pessoal ou na família. Quando questionados sobre uso de álcool e outras drogas, $69,70 \%$ negaram uso pessoal ou na família.

Entre os que apresentaram TMC (n=69), maior parte não possuía plano de saúde; relatou não possuir doença crônica; não faziam uso de medicamento controlado; referiram não apresentar diagnóstico prévio e confirmado de TMC individualmente ou em alguém da família; não fazia uso de álcool e outras drogas individualmente ou alguém da família.
A Tabela 4 traz as variáveis que se mantiveram associadas estatisticamente $(\mathrm{p}<0,05)$ à presença de transtorno mental nos adultos investigados.

Tabela 4 - Variáveis Sociodemográficas, Condições de Vida e Condições de Saúde que Apresentaram Associação Positiva com os Transtornos Mentais Comuns (escore $\geq 7$ SRQ-20

\begin{tabular}{|c|c|c|c|}
\hline Variáveis & $\begin{array}{c}<7 \text { SRQ-20 } \\
\mathbf{n}(\%)\end{array}$ & $\begin{array}{c}\geq 7 \text { SRQ-20 } \\
\mathbf{n}(\%)\end{array}$ & p-value \\
\hline \multicolumn{3}{|l|}{ Sexo } & 0,001 \\
\hline Feminino & $113(69,33)$ & $50(30,67)$ & \\
\hline Masculino & $115(85,82)$ & $19(14,18)$ & \\
\hline \multicolumn{3}{|l|}{ Estado Civil } & 0,002 \\
\hline União Estável & $7(46,67)$ & $8(53,33)$ & \\
\hline Casado(a) & $107(82,31)$ & $23(17,69)$ & \\
\hline Separado(a) & $5(50,00)$ & $5(50,00)$ & \\
\hline Viúvo(a) & $10(58,82)$ & $7(41,18)$ & \\
\hline Solteiro(a) & $99(79,20)$ & $26(20,80)$ & \\
\hline \multicolumn{3}{|l|}{ Anos de estudos } & 0,000 \\
\hline 0 a 4 & $35(54,69)$ & $29(45,31)$ & \\
\hline 5 a 8 & $58(85,29)$ & $10(14,71)$ & \\
\hline 9 ou mais & $135(81,82)$ & $30(18,18)$ & \\
\hline \multicolumn{3}{|c|}{ Uso de medicamento controlado } & 0,002 \\
\hline Sim & $39(61,90)$ & $24(38,10)$ & \\
\hline Não & $189(80,77)$ & $45(19,23)$ & \\
\hline \multicolumn{3}{|c|}{$\begin{array}{l}\text { Diagnóstico prévio/confirmado de TMC no } \\
\text { indivíduo ou alguém na família }\end{array}$} & 0,000 \\
\hline Sim & $34(57,63)$ & $25(42,37)$ & \\
\hline Não & $194(81,51)$ & $44(18,49)$ & \\
\hline
\end{tabular}

A prevalência dos TMC foi significativamente maior ( $\mathrm{p}$ $<0,05)$ nos adultos do sexo feminino, em união estável, com 0 a 4 anos de estudos, que faziam uso de medicamento controlado, e que informaram diagnóstico prévio/confirmado de TMC pessoal ou na família.

\section{DISCUSSÃO}

A prevalência de TMC evidenciada neste estudo $(23,2 \%)$ foi semelhante àquelas encontradas em outras pesquisas (Rocha et al., 2010; Morais \& Segri, 2011; Lucchese et al., 2014).

Em relação aos dados sociodemográficos dos adultos com TMC apresentados aqui, estudos nacionais e internacionais corroboram estes achados, evidenciando maiores prevalências em mulheres adultas jovens, solteiras.

Entretanto, houve diferença quanto ao nível de escolaridade, em que as demais pesquisas encontraram maior prevalência em pessoas com baixo nível e este estudo 
mostrou o contrário (Silva \& Menezes, 2008; Rocha et al., 2010; Silva et al., 2010; Estevam, Marcon, Antonio, Munari \& Waidman, 2011; Morais \& Segri, 2011; Lucchese et al., 2014).

Foi evidenciado que a renda mensal familiar dos portadores de TMC era, em sua maioria, de até 1 salário mínimo. Sobre isso, autores discorrem que a baixa renda, geralmente, está associada à dificuldade de inserção no mercado de trabalho, à pouca valorização e à condições de vida incertas, podendo ser considerada raiz de outros problemas sociais que induzem a má qualidade de vida e, consequentemente, problemas psicológicos futuros.

Em relação às demais condições de vida e às condições de saúde, outras pesquisas encontraram resultados semelhantes (Morais \& Segri, 2011; Rocha et al., 2010; Lucchese et al., 2014; Silva et al., 2010; Silva \& Menezes, 2008; Estevam et al., 2011). Apenas na variável "uso de álcool e outras drogas do indivíduo e/ou alguém na família”, em que maioria desta amostra negou, foi possível identificar divergência com outra pesquisa realizada no Acre, região Norte do Brasil, que encontrou maior prevalência de TMC nos indivíduos que faziam consumo de álcool (Silva, Corradi-Webster, Donato, Hayashida \& Siqueira, 2014).

$\mathrm{Na}$ análise dos fatores associados, quanto à variável sexo feminino apresentar-se com maior prevalência do desfecho, outros estudos confirmam este achado. A explicação para isso se deve às desvantagens das mulheres com a dupla jornada de trabalho, baixos salários e aspectos relacionados à reprodução, pois a mulher que vivencia o trabalho e o encargo familiar, geralmente, renuncia ao próprio cuidado para dedicar-se ao próximo, culminando em quadros de consternação, ansiedade, frustração, angústia, adoecimento e, sobretudo, ocorrência de transtornos mentais.

Além disso, as mulheres percebem facilmente o adoecimento, expressam prontamente seus sintomas e informam com maior frequência problemas de saúde, quando comparadas aos homens (Morais \& Segri, 2011; Rocha et al., 2010; Santos et al., 2010; Murcho et al., 2016).

Ao analisar a situação conjugal, os resultados revelaram associação no nível univariado dos TMC com a condição de união estável. Este achado diverge do encontrado por outro estudo, o qual mostrou associação de proteção com a condição de ser casado e associação de vulnerabilidade com ser solteiro ou viúvo (Rocha et al., 2010).
Apresentar zero a quatro anos de estudos se mostrou associado aos TMC, conforme esperado, uma vez que estudos já evidenciaram que a baixa escolaridade é um prenunciador importante de vulnerabilidade para o desenvolvimento dos TMC (Rocha et al., 2010; Carlotto et al., 2011; Silva \& Menezes, 2008).

Observou-se, ainda, associação do uso de medicamentos controlados com TMC nos adultos estudados. Explicação para esta associação advém do fato de que, possivelmente, ao procurarem o serviço de saúde, muitos usuários apresentam queixas somáticas inespecíficas, assim, há uma dificuldade no manejo correto dessas manifestações de sofrimento, levando ao subdiagnóstico e prescrição abusiva de benzodiazepínicos, exames e encaminhamentos sem necessidade (Silva $\&$ Menezes, 2008).

Mesmo que a maioria da amostra não referiu presença de doença crônica, autores discutem que outra explicação para a associação entre uso de medicamento controlado e TMC se refere ao fato de que na ESF é comum encontrar adultos portadores de doenças crônicas como hipertensão e diabetes, revelando a existência mais alta do TMC quando avaliadas populações que necessitam de acompanhamento de saúde por doença crônica, possivelmente, devido às limitações impostas pela doença (Silva \& Menezes, 2008).

Quanto à associação entre TMC aferido pelo SRQ-20 e diagnóstico confirmado de TMC no indivíduo ou na família, poucos estudos discutem esta relação. Sobre isso, os autores debatem que, muitas vezes, a família não conhece o significado da doença e isso leva os familiares a se sentirem perdidos em relação ao cuidado com o doente e isolados pelos serviços de saúde, agravando quadros de ansiedade ou depressão.

Assim, uma maior atenção para detecção de sintomas de comprometimento da saúde mental dos adultos e de seus familiares deve ser tomada, estabelecendo assistência integrada, capaz de satisfazer às reais necessidades do doente e da família para o reestabelecimento da saúde, minimizando o sofrimento e melhorando a qualidade de vida (Gluloum et al., 2011; Estevam et al., 2011).

Reforça-se, também, o fato das questões "Diagnóstico prévio/confirmado de TMC no indivíduo ou alguém na família" e "Uso de álcool e outras drogas do indivíduo e/ou alguém na família” apresentarem limitações nos resultados, uma vez que é importante reconhecer que o impacto de cada uma destas situações é diferente se estiver presente no próprio ou no familiar. 
Neste estudo, não foram incluídas outras questões sobre os estilos de vida das pessoas, na intenção de manter um questionário não extenso, uma vez que na etapa de pré-teste, alguns participantes já haviam sinalizado acerca do tempo para respostas. Contudo, reconhece-se como uma possível limitação do estudo, pois os estilos de vida podem se constituir como fatores promotores ou protetores da TMC.

Esperava-se que outras variáveis se mantivessem associadas ao desfecho ao nível univariado, tais como número de filhos, ter cônjuge falecido há mais de 5 anos, apresentar doenças crônicas, fazer uso de álcool ou outras drogas e renda mensal. Entretanto, a frequência do desfecho nas categorias de algumas variáveis explicativas foi baixa, dificultando as comparações.

Além disso, a maneira como as variáveis foram avaliadas e categorizadas pode ter influenciado os resultados, mesmo que a decisão pela categorização tenha sido embasada em outros estudos que avaliaram TMC em adultos na tentativa de facilitar as comparações (Rocha et al., 2010; Carlotto et al., 2011; Pie et al., 2013).

\section{CONCLUSÕES}

Observou-se elevada prevalência de TMC, sendo corroborada por outros estudos nacionais e internacionais. Mantiveram-se associadas, no nível univariado, as variáveis sexo feminino; estar e união estável; apresentar 0 a 4 anos de estudos; fazer uso de medicamento controlado; apresentar diagnóstico prévio/confirmado de TMC pessoal ou em alguém na família.

É importante reforçar que os resultados deste estudo devem ser avaliados com cautela em função das possíveis limitações da pesquisa. Uma delas se deve ao tipo de estudo. Por ser se tratar de um estudo transversal, não é possível concluir relações causais, devido ao fato de não evidenciar uma relação de temporalidade e causalidade entre as variáveis envolvidas. Assim, não se pode descartar a hipótese de causa reversa, ou seja, não é possível identificar se os TMC influenciaram os fatores associados ou vice-versa, principalmente nos casos que faziam uso de medicamento controlado e que referiram diagnóstico confirmado de TMC.

Os resultados apresentados evidenciaram que as características associadas são passíveis de serem investigadas e acompanhadas na prática clínica e cotidiana. Destaca-se, portanto, a necessidade de qualificação dos profissionais da Atenção Primária à Saúde, visando à detecção e acolhimento das pessoas com TMC, tendo em vista a "invisibilidade" destes transtornos devido à ausência de sinais e sintomas mais severos.
Ações de educação direcionadas à promoção da saúde e à uma assistência integral, com a efetivação da saúde mental dos usuários em todos os programas já existentes na Estratégia Saúde da Família, devem ser implementadas.

Por fim, é preciso enfatizar o acolhimento, a escuta qualificada, o trabalho com grupos, o cuidado no tratamento e no uso de medicamentos diversos e o foco na família, fortalecendo as redes de apoio e instituindo ações intersetoriais no cuidado aos usuários com TMC, no sentindo de promover saúde e melhorar a qualidade de vida desta população.

\section{REFERÊNCIAS BIBLIOGRÁFICAS}

Carlotto, M. S., Amazarray, M. R., Chinazzo, I., \& Taborda, L. (2011). Common mental disorders and associated factors among workers: An analysis from a gender perspective. Cadernos de Saúde Coletiva, 19(2), 172-178.

Chandra, P. S., \& Satyanarayana, V. A. (2010). Gender disadvantage and common mental disorders in women. International Review of Psychiatry, 22(5), 513-524.

Coutinho, M. P. L., Rodrigues, I. F., \& Ramos, N. (2012). Transtornos mentais comuns no contexto migratório internacional. PSICO, 43(3), 400-407.

Estevam, M. C., Marcon, S. S., Antonio, M. M., Munari, D. B., \& Waidman, M. A. P. (2011). Living with mental disorders: Family members' perspective have on primary care. Revista da Escola de Enfermagem da USP, 45(3), 679-686. doi: 10.1590/S0080- 62342011000300019

Fonseca, M. L. G., Guimarães, M. B. L., \& Vasconcelos, E. M. (2008). Sofrimento difuso e transtornos mentais comuns: Uma revisão bibliográfica. Revista APS, 11(3), 285-294.

Franken, I., Coutinho, M. P. L., \& Ramos, M. N. P. (2012). Representações sociais, saúde mental e imigração internacional. Psicologia: Ciência e Profissão, 32(1), 202-219. doi: 10.1590/S1414-98932012000100015

Gluloum, S., Bener, A., \& Abou-Salen, M. T. (2011). Prevalence of mental disorders in adult population attending primary health care setting in Qatari population. Journal of Pakistan Medical Association, 61(3), 216-221. 
Gonçalves, D. M., Tetelbon, S. A., \& Kapczinski, F. (2008). Avaliação de desempenho do Self-Reporting Questionnaire como instrumento de rastreamento psiquiátrico: Um estudo comparativo com o Structured Clinical Interview for DSM-IV-TR. Cadernos de Saúde Pública, 24(2), 380-390. doi: 10.1590/S0102311X2008000200017

Gonçalves, D. M., \& Kapczinski, F. (2008). Transtornos mentais em comunidade atendida pelo Programa Saúde da Família. Cadernos de Saúde Pública, 24(7), 1641-1650.

Jansen, K., Mondin, T. C., Ores, L. C., Souza, L. D. M., Konradt, C. E., Pinheiro, R. T., \& Silva, R. A. (2011). Transtornos mentais comuns e qualidade de vida em jovens: Uma amostra populacional de Pelotas, Rio Grande do Sul, Brasil. Cadernos de Saúde Pública, 27(3), 65-79.

Jenkins, R., Mbatia, J., Singleton, N., \& White, B. (2010). Common mental disorders and risk factors in urban Tanzania. International Journal of Environmental Research and Public Health, 7(6), 2543-2558. doi: 10.3390/ijerph706254

Lucchese, R. S. K., Bonfin, S. P., Vera, I., \& Santana, F. R. (2014). Prevalência de transtorno mental comum na atenção primária. Acta Paulista de Enfermagem, 27(3), 200-207. doi: 10.1590/1982-0194201400035

Ministério da Saúde. Secretaria de vigilância em saúde. (1998). Portaria no 344, de 12 de maio de 1998. Brasília: Ministério da Saúde.

Morais, M. L. S., \& Segri, N. J. (2011). Prevalência de transtornos mentais comuns auto-referidos e sua relação com os serviços de saúde em municípios da Baixada Santista - SP. BIS. Boletim do Instituto de Saúde, 13(2), 141-146.

Murcho, N., Pacheco, E., \& Jesus, S. N. (2016). Transtornos mentais comuns nos Cuidados de Saúde Primários: Um estudo de revisão. Revista Portuguesa de Enfermagem de Saúde Mental (15), 30-36. doi: 10.19131/ rpesm.0129

Pagano, M., \& Gauvreau, K. (2011). Princípios de bioestatística. São Paulo: Pioneira Thomson Learning.
Pie, A. C. S., Pinto, L. L. T., Rocha, S. V., Cardoso, J. P., Amorim, C. R., \& Carneiro, L. R. V. (2013). Nível de atividade física e transtornos mentais comuns entre trabalhadores de uma instituição de ensino superior da Bahia. Arquivo de Ciências do Esporte, 1(1), 46-53.

Portugal, F. B., Campos, M. R., Gonçalves, D. A., Mari, J. J., \& Fortes, S. L. C. L. (2016). Qualidade de vida em pacientes da atenção primária do Rio de Janeiro e São Paulo, Brasil: Associações com eventos de vida produtores de estresse e saúde mental. Ciência \& Saúde Coletiva, 21(2), 497-508.

Rocha, S. V., Almeida, M. M. G., Araújo, T. M., \& Virtuoso, J. J. S. (2010). Prevalência de transtornos mentais comuns entre residentes em áreas urbanas de Feira de Santana, Bahia. Revista Brasileira de Epidemiologia, 13(4), 630-640. doi: 10.1590/S1415-790X2010000400008

Santos, K. O. B., Araújo, T. M., Pinho, P. S., \& Silva, A. C. C. (2010). Avaliação de um instrumento de mensuração de morbidade psíquica: Estudo de validação do self-reportingquestionnaire (SRQ-20). Revista Baiana de Saúde Pública, 34(3), 544-560.

Silva, A. T. C., \& Menezes, P. R. (2008). Esgotamento profissional e transtornos mentais comuns em agentes comunitários de saúde. Revista de Saúde Pública, 42(5), 921-929. doi: 10.1590/S0034-89102008000500019

Silva, B. P., Corradi-Webster, C. M., Donato, E. C. S. G., Hayashida, M., \& Siqueira, M. M. (2014). Transtornos mentais comuns e consumo de bebida alcoólica e tabaco entre estudantes de enfermagem de uma universidade pública na Amazônia Ocidental brasileira. SMAD. Revista Eletrônica Saúde Mental Álcool e Drogas, 10(2), 93-100. doi: 10.11606/issn.1806-6976.v10i2p93-100

Silva, R. A., Ores, L. C., Mondin, T. C., Rizzo, R. N., Moraes , I. G. S., Jansen, K., \& Pinheiro, R. T. (2010). Transtornos mentais comuns e auto-estima na gestação: Prevalência e fatores associados. Cadernos de Saúde Pública, 26(9), 1832-1838.

Skapinakis, P., Bellos, S., Koupidis, S., Grammatikopoulos, L., Theodorakis, P. N., \& Mavreas, V. (2013). Prevalence and sociodemographic associations of common mental disorders in a nationally representative sample of the general population of Greece. BMC Psychiatry, 4(13), 163. doi: 10.1186/1471-244X-13-163 